\title{
Classical and quantum mechanics of the nonrelativistic Snyder model in curved space
}

\author{
S. Mignemi ${ }^{\dagger}$ \\ Dipartimento di Matematica, Università di Cagliari \\ viale Merello 92, 09123 Cagliari, Italy \\ and INFN, Sezione di Cagliari
}

\begin{abstract}
The Snyder-de Sitter (SdS) model is a generalization of the Snyder model to a spacetime background of constant curvature. It is an example of noncommutative spacetime admitting two fundamental scales besides the speed of light, and is invariant under the action of the de Sitter group. Here we consider its nonrelativistic counterpart, i.e. the Snyder model restricted to a three-dimensional sphere, and the related model obtained by considering the anti-Snyder model on a pseudosphere, that we call anti-Snyder-de Sitter (aSdS).

By means of a nonlinear transformation relating the SdS phase space variables to canonical ones, we are able to investigate the classical and the quantum mechanics of a free particle and of an oscillator in this framework. As in their flat space limit, the SdS and aSdS models exhibit rather different properties. In the SdS case, a lower bound on the localization in position and momentum space arises, which is not present in the aSdS model. In the aSdS case, instead, a specific combination of position and momentum coordinates cannot exceed a constant value.

We explicitly solve the classical and the quantum equations for the motion of the free particle and of the harmonic oscillator. In both the SdS and aSdS cases, the frequency of the harmonic oscillator acquires a dependence on the energy.
\end{abstract}

P.A.C.S. Numbers: 02.40.Gh; 45.20.Jj; 03.65.Ca.

$\dagger$ e-mail: smignemi@unica.it 


\section{INTRODUCTION}

Already in 1947, in the attempt to introduce a short distance cutoff in field theory, Snyder proposed a model of noncommutative spacetime, admitting a fundamental length scale [1]. In spite of the presence of a fundamental length, the model was invariant under the action of the Lorentz group. Snyder's proposal was revived in recent years, when considerations on quantum gravity and string theory suggested that the structure of spacetime may be noncommutative at scales close to the Planck length [2]. Noncommutativity of spacetime can in fact account for the modification of the Heisenberg uncertainty relations [3] and the existence of a minimal bound on the localization of particles in spacetime [4] that follow from any quantum theory of spacetime. Actually, it has been argued that already at the Newtonian level the action of gravity calls for a modification of the uncertainty relations [5].

Similar arguments suggest that any theory of quantum gravity should require the introduction of a fundamental observer-independent scale with the physical dimension of an energy, in addition to the speed of light. The new fundamental energy scale sets a bound on the allowed values of the momentum of the elementary particles, and gives rise to a deformation of the Poincaré symmetry and of the energy-momentum dispersion relations. This line of thought, which is at the basis of Doubly Special Relativity (DSR) [6], is in some sense dual to that leading to noncommutative geometry, and in fact the natural spacetime background for DSR is noncommutative.

In particular, the Snyder model can be interpreted as an example of DSR [7], and its dynamics has been investigated in this context in [8]. Further aspects of the Snyder model were studied in [9].

Pursuing the DSR idea, it was later proposed that the cosmological constant $\Lambda$ could be introduced as a third fundamental observer-independent parameter in the algebra of spacetime symmetries [10]. The model based on these assumptions was called Triply Special Relativity (TSR), because it is based on three fundamental parameters. In the following, we shall usually adopt the more modest denomination Snyder-de Sitter (SdS) model, especially when dealing with the nonrelativistic limit.

The proposal of TSR was justified by considerations arising from quantum gravity [10]. From a more formal point of view, its interest relies on the fact that both spacetime and momentum space are noncommutative, and on its symmetry for the interchange of positions and momenta, that gives rise to minimal uncertainties for both of them. In spite of its complexity, the model can be solved exactly in some simple cases, as we shall show.

TSR is based on the algebra generated by the positions $x_{\mu}$, momenta $p_{\mu}$ and Lorentz generators $J_{\mu \nu}$. The Lorentz generators satisfy the usual commutation relations of the Lorentz algebra, together with

$$
\left[J_{\mu \nu}, x_{\lambda}\right]=i \hbar\left(\eta_{\mu \lambda} x_{\nu}-\eta_{\nu \lambda} x_{\mu}\right), \quad\left[J_{\mu \nu}, p_{\lambda}\right]=i \hbar\left(\eta_{\mu \lambda} p_{\nu}-\eta_{\nu \lambda} p_{\mu}\right),
$$

while

$$
\begin{gathered}
{\left[x_{\mu}, x_{\nu}\right]=i \hbar \beta^{2} J_{\mu \nu}, \quad\left[p_{\mu}, p_{\nu}\right]=i \hbar \alpha^{2} J_{\mu \nu},} \\
{\left[x_{\mu}, p_{\nu}\right]=i \hbar\left[\eta_{\mu \nu}+\alpha^{2} x_{\mu} x_{\nu}+\beta^{2} p_{\mu} p_{\nu}+\alpha \beta\left(x_{\mu} p_{\nu}+p_{\mu} x_{\nu}-J_{\mu \nu}\right)\right],}
\end{gathered}
$$


with $\mu, \nu=0, \ldots, 3$. The $J_{\mu \nu}$ and $p_{\mu}$ generate a de Sitter or anti-de Sitter subalgebra (depending on the sign of $\alpha^{2}$ ) that describes the spacetime symmetries of the model. As in DSR, the action of the translations on the spatial coordinates is nonlinear. The coupling constants $\alpha$ and $\beta$ have dimension of inverse length and inverse mass, respectively. ${ }^{1}$ They are usually identified with the square root of the cosmological constant, $\alpha \sim 10^{-24} \mathrm{~cm}^{-1}$, and with the inverse of the Planck mass, $\beta \sim 10^{5} \mathrm{~g}^{-1}$, but larger scales compatible with observations may be chosen. In the following, we require $\alpha \beta \ll 1 / \hbar$. With the previous identifications, this inequality is verified by almost 60 orders of magnitude. The limit $\alpha \rightarrow 0$ gives the flat Snyder model, while the limit $\beta \rightarrow 0$ yields the Heisenberg algebra of quantum mechanics in a de Sitter background endowed with projective coordinates.

From (1.1) it follows that in TSR both position and momentum components do not commute among themselves. For what concerns momenta, this is typical of curved spacetimes, while, as we have seen, the noncommutativity of positions characterizes Snyder spaces. Also notable is the duality of the model for position and momentum interchange through $x_{\mu} \leftrightarrow p_{\mu}, \alpha \leftrightarrow \beta$. Moreover, as was recognized in [11], TSR can be viewed as a nonlinear realization of a model introduced by Yang [12] soon after Snyder's paper, based on the conformal group $\mathrm{SO}(1,5)$. The commutation relations of Yang's model are again those of the Lorentz algebra, together with

$$
\begin{gathered}
{\left[x_{\mu}, x_{\nu}\right]=i \hbar \beta^{2} J_{\mu \nu}, \quad\left[p_{\mu}, p_{\nu}\right]=i \hbar \alpha^{2} J_{\mu \nu}, \quad\left[x_{\mu}, p_{\nu}\right]=i \hbar \eta_{\mu \nu} F} \\
{\left[x_{\mu}, F\right]=-i \hbar \beta^{2} p_{\mu}, \quad\left[p_{\mu}, F\right]=i \hbar \alpha^{2} x_{\mu}}
\end{gathered}
$$

where a central charge $F$ that commutes with the Lorentz generators has been introduced. Although in a quantum setting the linear realization (1.3) can be more convenient, it is not suitable for an implementation in classical phase space, and we shall not consider it further.

TSR can also be derived from a six-dimensional model, with the higher-dimensional position and momentum variables satisfying quadratic constraints [13]. Other aspects of the theory have been studied in $[14,15]$.

Recently, the kinematics and the dynamics of the Snyder model have been investigated in its three-dimensional (nonrelativistic) version both for classical and quantum systems $[16]{ }^{2}$ exploiting the existence of a nonlinear map from canonical to Snyder's phase space. The relevance of the nonrelativistic limit is given by the fact that it exhibits all the essential features of the original model, but avoids the complications related to relativistic dynamics. These investigations evidentiated that the physical properties of the model depend on the sign of the coupling constant appearing in its algebra. For positive coupling constant, the momenta are allowed to take any real value, but in the quantum theory a minimal uncertainty in the positions arises. On the contrary, in the case of negative coupling constant, that was called anti-Snyder, the momentum is bounded, but no minimal uncertainty for positions occurs in the quantum theory.

1 We adopt units in which $c=1$. Then $\hbar \sim 10^{-38} \mathrm{~g} \mathrm{~cm}$.

2 For a different approach see [17]. 
In this paper, we extend the investigations of ref. [16] to the case of the nonrelativistic Snyder model in a constant curvature background. We carry out this task by exploiting the possibility to define a simple linear (but of course not canonical) transformation that relates the SdS algebra to Snyder's. We show that, like in the case of the flat Snyder model, the physics depends strongly on the sign of the coupling constants $\alpha^{2}$ and $\beta^{2}$ in (1.2). In order to obtain a consistent algebra, both constants must have the same sign. If the sign is positive, the indetermination relations imply the existence of both a minimal length and a minimal momentum, while for negative sign no restriction arises.

In the first case, formal eigenvalues of position and momentum take discrete values. However, the existence of a minimal uncertainty implies that the position and momentum eigenstates cannot have a definite eigenvalue. It follows that the formal solutions of the eigenvalue equations are not physical, and in fact have diverging values of the uncertainties $\Delta x$ and $\Delta p$. In a similar situation, Kempf et al. [18] suggested that a modified eigenvalue equation should be introduced that give rise to states that minimize the uncertainty. In our case we are not able to find a suitable generalization of their equation, but discuss a class of functions that give an approximate solution to the problem.

No minimal uncertainties occur instead when $\alpha^{2}$ and $\beta^{2}$ are negative. In this case, however, a specific combination of spatial and momentum coordinates must satisfy an upper bound. This can be interpreted as a sign of a dependence of the geometry on the energy.

Another interesting application of our method is the study of the harmonic oscillator. In analogy with the flat Snyder model, both in classical and quantum mechanics its solution contains corrections of order $\left(\beta^{2}+\alpha^{2} / \omega_{0}^{2}\right) E$ to the standard case, with $E$ the energy and $\omega_{0}$ the classical frequency of the oscillator. In particular, the frequency of oscillation is no longer independent from the energy. Furthermore, in the aSdS case the spectrum of energy contains only a finite number of states.

\section{CLASSICAL MECHANICS}

We first consider the implementation of the nonrelativistic SdS model in classical phase space. The model is defined on a three-dimensional space of constant curvature with Euclidean signature. Its phase space is endowed with a noncanonical symplectic structure given by the classical limit of the commutator algebra (1.2). We study the kinematics and some simple examples of dynamics in one dimension.

\subsection{The model}

In classical mechanics, the motion in the nonrelativistic SdS model can be described by postulating a noncanonical symplectic structure on phase space, with fundamental Poisson brackets [13]

$$
\begin{aligned}
& \left\{x_{i}, x_{j}\right\}=\beta^{2} J_{i j}, \quad\left\{p_{i}, p_{j}\right\}=\alpha^{2} J_{i j}, \\
& \left\{x_{i}, p_{j}\right\}=\delta_{i j}+\alpha^{2} x_{i} x_{j}+\beta^{2} p_{i} p_{j}+2 \alpha \beta p_{i} x_{j},
\end{aligned}
$$

where $i, j \ldots=1,2,3$. The Poisson brackets (2.1) are obtained from (1.2) by introducing the standard expression $J_{i j}=x_{i} p_{j}-x_{j} p_{i}$ for the generators of the rotations. 
In spite of the notation, we allow $\alpha^{2}$ and $\beta^{2}$ to be negative, but, in order for the Jacobi identities to hold, both $\alpha^{2}$ and $\beta^{2}$ must have the same sign. ${ }^{3}$ The case of positive coupling constants corresponds to the Snyder model on a spherical background, while the model with negative $\alpha^{2}$ and $\beta^{2}$ gives rise to the anti-Snyder model on a pseudosphere. We call the latter anti-Snyder-de Sitter (aSdS) model.

The Poisson brackets (2.1) can be obtained from those of the flat Snyder model, with position variables $\mathcal{X}_{i}$ and momentum variables $\mathcal{P}_{i}$ obeying

$$
\left\{\mathcal{X}_{i}, \mathcal{X}_{j}\right\}=\beta^{2}\left(\mathcal{X}_{i} \mathcal{P}_{j}-\mathcal{X}_{j} \mathcal{P}_{i}\right), \quad\left\{\mathcal{P}_{i}, \mathcal{P}_{j}\right\}=0, \quad\left\{\mathcal{X}_{i}, \mathcal{P}_{j}\right\}=\delta_{i j}+\beta^{2} \mathcal{P}_{i} \mathcal{P}_{j}
$$

by performing a linear unimodular but non-symplectic transformation of the phase space coordinates,

$$
x_{i}=\mathcal{X}_{i}+\frac{\beta}{\alpha} \lambda \mathcal{P}_{i}, \quad p_{i}=(1-\lambda) \mathcal{P}_{i}-\frac{\alpha}{\beta} \mathcal{X}_{i},
$$

where $\lambda$ is a free parameter, that can be chosen arbitrarily. Actually, the freedom in the choice of a parameter $\lambda$ is already present in the flat Snyder model. In that case, the symplectic transformations $\mathcal{X}_{i} \rightarrow \mathcal{X}_{i}+\lambda \mathcal{P}_{i}, \mathcal{P}_{i} \rightarrow \mathcal{P}_{i}$ leaves the Poisson brackets (2.2) invariant. Analogously, the transformation from the representation (2.3) of the SdS model with $\lambda=0$ to one with $\lambda \neq 0$ is symplectic. The physical results are therefore independent of the choice of $\lambda$.

In ref. [16] it has been shown that the phase space variables $\mathcal{X}_{i}$ and $\mathcal{P}_{i}$ can in turn be written in terms of coordinates $X_{i}, P_{i}$ that satisfy canonical Poisson brackets, by means of the nonlinear transformation

$$
\mathcal{P}_{i}=\frac{P_{i}}{\sqrt{1-\beta^{2} P_{k}^{2}}}, \quad \mathcal{X}_{i}=\sqrt{1-\beta^{2} P_{k}^{2}} X_{i}
$$

Combining (2.3) and (2.4), the coordinates $x_{i}$ and $p_{i}$ that satisfy the SdS Poisson brackets can be written in terms of canonical coordinates $X_{i}, P_{i}$, as

$$
\begin{aligned}
& x_{i}=\sqrt{1-\beta^{2} P_{k}^{2}} X_{i}+\lambda \frac{\beta}{\alpha} \frac{P_{i}}{\sqrt{1-\beta^{2} P_{k}^{2}}}, \\
& p_{i}=-\frac{\alpha}{\beta} \sqrt{1-\beta^{2} P_{k}^{2}} X_{i}+(1-\lambda) \frac{P_{i}}{\sqrt{1-\beta^{2} P_{k}^{2}}},
\end{aligned}
$$

with inverse transformations

$$
X_{i}=\frac{(1-\lambda) \alpha x_{i}-\lambda \beta p_{i}}{\alpha \sqrt{1+\left(\alpha x_{k}+\beta p_{k}\right)^{2}}}, \quad P_{i}=\frac{\beta p_{i}+\alpha x_{i}}{\beta \sqrt{1+\left(\alpha x_{k}+\beta p_{k}\right)^{2}}}
$$

It is important to note that if $\alpha^{2}, \beta^{2}<0$, one must impose $\left|\alpha x_{k}+\beta p_{k}\right|<1$. The range of definition of the coordinates depends therefore on the value of the momentum. A possible interpretation of this fact is that the radius of the pseudosphere is a function of the momentum of the particle: this situation is common in DSR theories defined in curved spaces, where the metric properties are momentum dependent $[19,14]$.

3 In this paper, we adopt the following convention: when $\alpha^{2}$ and $\beta^{2}$ are negative, also $\alpha \beta$ is negative, while for expressions linear in $\alpha$ and $\beta$ we define $\alpha=\sqrt{\left|\alpha^{2}\right|}, \beta=\sqrt{\left|\beta^{2}\right|}$. 


\subsection{Symmetries}

The full phase space is invariant under the $S O(5)$ or $S O(4,1)$ group, depending on the sign of the coupling constants. However, from a physical point of view, the symmetries of the configuration space are more interesting. These are of course described by the groups $S O(4)$ or $S O(3,1)$, that leave invariant a three-dimensional space of constant positive (resp. negative) curvature, and are generated by the angular momentum $J_{i j}=x_{i} p_{j}-x_{j} p_{i}$ and the momentum $p_{i}$.

The phase-space coordinates transform as vectors under the action of the generators of the rotations $J_{i j}$,

$$
\left\{J_{i j}, x_{k}\right\}=\delta_{i k} x_{j}-\delta_{i j} x_{k}, \quad\left\{J_{i j}, p_{k}\right\}=\delta_{i k} p_{j}-\delta_{i j} p_{k},
$$

The symmetry under rotations is therefore realized in the usual way.

The action of the translation generators $p_{i}$ on phase-space variables can be read from (2.1). While the momenta transform according to the standard law for a constant curvature space, the action of the translations on the position coordinates is deformed and is nonlinear.

Due to the Jacobi identities, the fundamental Poisson brackets (2.1) transform covariantly under the action of the symmetry group.

\subsection{Classical motion}

The Hamiltonian for a free particle can be defined in the usual way as

$$
H=\frac{p_{i}^{2}}{2 m}
$$

The Hamilton equations are obtained taking into account the deformed Poisson structure, and read

$$
\dot{x}_{i}=\left(1+\beta^{2} p_{k}^{2}+2 \alpha \beta x_{k} p_{k}\right) p_{i}+\alpha^{2} x_{k} p_{k} x_{i}, \quad \dot{p}_{i}=-\alpha^{2}\left(p_{k}^{2} x_{i}-x_{k} p_{k} p_{i}\right) .
$$

The relation between velocity and momentum is no longer linear, and the equations are difficult to solve. Nevertheless, the solution can easily be obtained in one dimension. In this case, the Poisson brackets reduce to

$$
\{x, p\}=1+(\alpha x+\beta p)^{2},
$$

and the Hamilton equations read

$$
\dot{x}=\left[1+(\alpha x+\beta p)^{2}\right] \frac{p}{m}, \quad \dot{p}=0 .
$$

Hence the momentum $p$ is constant, $p=p_{0}$, and integration of the first equation yields

$$
x=\frac{1}{\alpha} \tan \frac{\alpha p_{0}}{m} t-\frac{\beta}{\alpha} p_{0}, \quad \text { if } \alpha^{2}>0,
$$




$$
x=\frac{1}{\alpha} \tanh \frac{\alpha p_{0}}{m} t-\frac{\beta}{\alpha} p_{0}, \quad \text { if } \alpha^{2}<0 .
$$

Notice that in the last case $|\alpha x+\beta p|<1$, as required.

A more interesting example of dynamics is the one-dimensional harmonic oscillator, with Hamiltonian

$$
H=\frac{p^{2}}{2 m}+\frac{m \omega_{0}^{2} x^{2}}{2} .
$$

Let us first consider the case $\alpha^{2}, \beta^{2}>0$. For unit mass, the Hamilton equations read

$$
\dot{x}=\left[1+(\alpha x+\beta p)^{2}\right] p, \quad \dot{p}=-\omega_{0}^{2}\left[1+(\alpha x+\beta p)^{2}\right] x .
$$

Using (2.13), it is easy to check that, like in standard classical mechanics, the Hamiltonian is conserved, namely

$$
\frac{p^{2}}{2}+\frac{\omega_{0}^{2} x^{2}}{2}=E,
$$

with $E$ the total energy of the oscillator.

The equations of motion can be solved as follows: define $z=\arctan (\alpha x+\beta p)$. From eqs. (2.13) it follows that

$$
\dot{z}=\alpha p-\omega_{0}^{2} \beta x .
$$

Deriving (2.15), and substituting (2.13), one obtains

$$
\ddot{z}=-\omega_{0}^{2} \frac{\sin z}{\cos ^{3} z},
$$

which admits the first integral

$$
\frac{1}{2} \dot{z}^{2}+\frac{\omega_{0}^{2}}{2} \tan ^{2} z=\omega_{0}^{2} \mathcal{E},
$$

with $\mathcal{E}$ an integration constant, proportional to $E$. These equations are identical to those of a classical particle moving in the effective potential $V=\omega_{0}^{2} \tan ^{2} z$, and have the same analytical form as those obtained in [16] for the flat Snyder model.

Integration of (2.17) yields

$$
z=\alpha x+\beta p=\frac{\sqrt{2 \mathcal{E}} \sin \omega t}{\sqrt{1+2 \mathcal{E} \cos ^{2} \omega t}}
$$

where $\omega=\sqrt{1+2 \mathcal{E}} \omega_{0}$. From (2.15) it is then easy to obtain

$$
\alpha p-\omega_{0}^{2} \beta x=\frac{\omega_{0} \sqrt{2 \mathcal{E}(1+2 \mathcal{E})} \cos \omega t}{\sqrt{1+2 \mathcal{E} \cos ^{2} \omega t}} .
$$

Substituting in (2.14), one finds the relation between $\mathcal{E}$ and $E$,

$$
\mathcal{E}=\gamma E, \quad \text { with } \quad \gamma=\beta^{2}+\frac{\alpha^{2}}{\omega_{0}^{2}},
$$


and then

$$
\omega=\sqrt{1+2 \gamma E} \omega_{0} .
$$

The solution can finally be written as

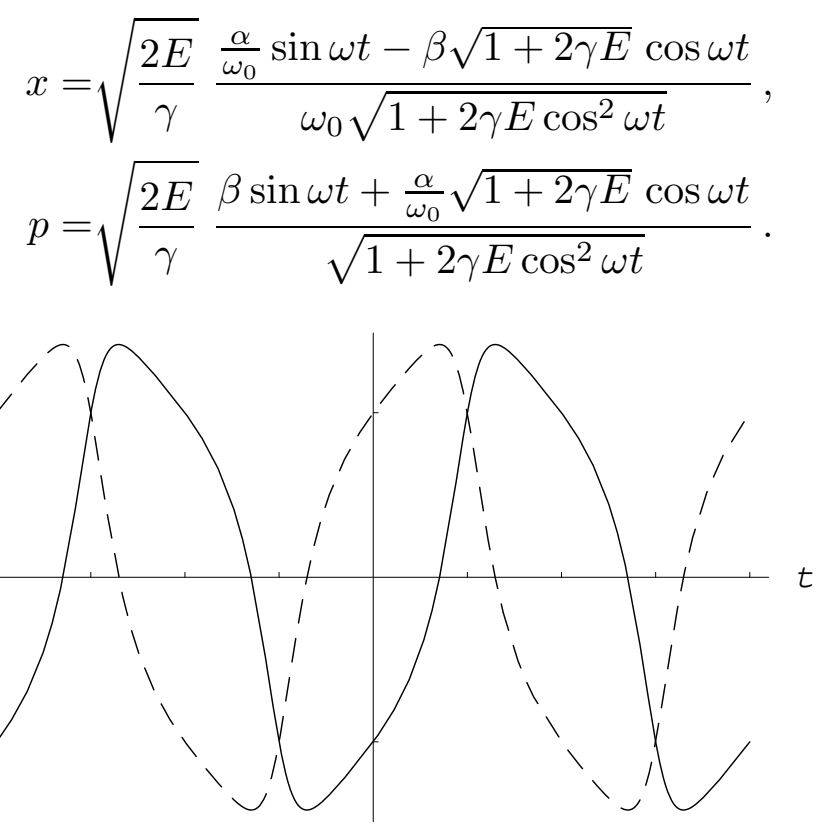

Fig. 1: The solution of the SdS oscillator for $\gamma E=3 / 2$. The solid line represents the coordinate $x$, the dashed line the coordinate $p$.

It appears that the solutions of the harmonic oscillator are periodic, but not sinusoidal, see Fig. 1. Moreover, their frequency depends on the energy of the oscillator. In order for the frequency to be real, the energy must be such that $1+2 \gamma E \geq 0$. This is always true if the energy is positive. The flat Snyder oscillator [16] is recovered in the limit $\alpha \rightarrow 0$.

Let us now consider the aSdS model. The calculations are essentially identical to the previous case, except that now one defines a variable $z=\operatorname{arctanh}(\alpha x+\beta p)$, that yields an effective potential $V=\omega_{0}^{2} \tanh z$. Proceeding as above, one recovers the relation (2.21) between frequency and energy (but now with negative $\gamma$ ) and

$$
\begin{aligned}
& x=\sqrt{\frac{2 E}{|\gamma|}} \frac{\frac{\alpha}{\omega_{0}} \sin \omega t-\beta \sqrt{1+2 \gamma E} \cos \omega t}{\omega_{0} \sqrt{1+2 \gamma E \cos ^{2} \omega t}}, \\
& p=\sqrt{\frac{2 E}{|\gamma|}} \frac{\beta \sin \omega t+\frac{\alpha}{\omega_{0}} \sqrt{1+2 \gamma E} \cos \omega t}{\sqrt{1+2 \gamma E \cos ^{2} \omega t}} .
\end{aligned}
$$

The character of the motion is similar to that found in the previous case, see Fig. 2. Again, one must impose the condition $0<E<-1 / 2 \gamma$ for the solutions to be real, that now gives rise to an upper bound for the energy, $2 E<\omega_{0}^{2} /\left(\left|\beta^{2}\right| \omega_{0}^{2}+\left|\alpha^{2}\right|\right)$, consistently with the bound $|\alpha x+\beta p|<1$. In the limit $\alpha \rightarrow 0$, this condition does not depend on the frequency and is the same that holds for the anti-Snyder model [16], while for $\beta \rightarrow 0$, gives $E<1 / 2\left|\beta^{2}\right|$, independently of $\omega_{0}$. 


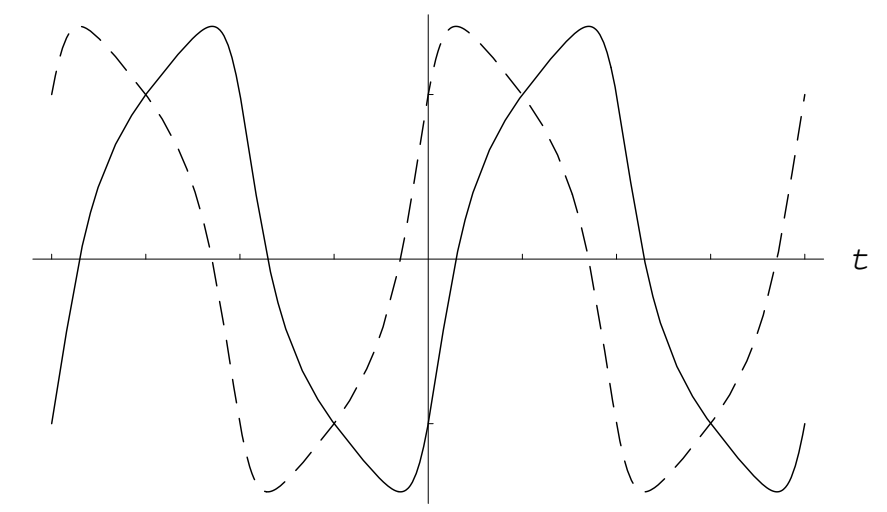

Fig. 2: The solution of the aSdS oscillator for $\gamma E=-3 / 8$. The solid line represents the coordinate $x$, the dashed line the coordinate $p$.

\section{QUANTUM KINEMATICS}

The map (2.5) can be exploited to construct the quantum operators for position and momentum that obey the SdS algebra. The deformation of the commutation relations implies a modification of the Heisenberg uncertainty relations and gives rise to minimal uncertainty for both position and momentum. The spectrum of the corresponding operators is changed accordingly. Modified uncertainty relations have been studied in different contexts in several papers $[3,18]$.

\subsection{Commutation relations and uncertainty}

For the (a)SdS model, the commutation relations (1.2) between the position operators $\hat{x}_{i}$ and the momentum operators $\hat{p}_{i}$ can be written in the equivalent form

$$
\begin{gathered}
{\left[\hat{x}_{i}, \hat{x}_{j}\right]=i \hbar \beta^{2} \hat{J}_{i j}, \quad\left[\hat{p}_{i}, \hat{p}_{j}\right]=i \hbar \alpha^{2} \hat{J}_{i j},} \\
{\left[\hat{x}_{i}, \hat{p}_{j}\right]=i \hbar\left[\delta_{i j}+\alpha^{2} \hat{x}_{i} \hat{x}_{j}+\beta^{2} \hat{p}_{i} \hat{p}_{j}+\frac{\alpha \beta}{2}\left(3 \hat{p}_{i} \hat{x}_{j}+\hat{x}_{j} \hat{p}_{i}-\hat{p}_{j} \hat{x}_{i}+\hat{x}_{i} \hat{p}_{j}\right)\right],}
\end{gathered}
$$

where the last equation is obtained from (1.2) by substiting the representation $\hat{J}_{i j}=$ $\frac{1}{2}\left(\hat{p}_{i} \hat{x}_{j}+\hat{x}_{j} \hat{p}_{i}-\hat{p}_{j} \hat{x}_{i}-\hat{x}_{i} \hat{p}_{j}\right)$ for the angular momentum. The lower degree of symmetry of (3.1) with respect to the classical expression (2.1) is due to operator ordering problems.

In the simple case in which $\left\langle\hat{p}_{i}\right\rangle=\left\langle\hat{x}_{i}\right\rangle=0$, the uncertainty relations that follow from (1.2) for states with vanishing angular momentum are

$$
\begin{aligned}
\Delta x_{i} \Delta p_{j} & \geq \frac{\hbar}{2}\left|\left\langle\left[\hat{x}_{i}, \hat{p}_{j}\right]\right\rangle\right|=\frac{\hbar}{2}\left|\delta_{i j}+\left\langle\left(\alpha \hat{x}_{i}+\beta \hat{p}_{i}\right)\left(\alpha \hat{x}_{j}+\beta \hat{p}_{j}\right)\right\rangle\right| \\
& \geq \frac{\hbar}{2}\left|\delta_{i j}+\alpha^{2} \Delta x_{i} \Delta x_{j}+\beta^{2} \Delta p_{i} \Delta p_{j}-\alpha \beta\left(\Delta x_{i} \Delta p_{j}+\Delta x_{j} \Delta p_{i}\right)\right|,
\end{aligned}
$$

where the Schwartz inequality has been used in the last step. In the one-dimensional case, the algebra (3.1) simplifies greatly. In particular,

$$
[\hat{x}, \hat{p}]=i \hbar\left[1+\alpha^{2} \hat{x}^{2}+\beta^{2} \hat{p}^{2}+\alpha \beta(\hat{x} \hat{p}+\hat{p} \hat{x})\right],
$$


and the uncertainty relations $(3.2)$ reduce to

$$
\Delta x \Delta p \geq \frac{\hbar}{2} \frac{\left|1+\alpha^{2}(\Delta x)^{2}+\beta^{2}(\Delta p)^{2}\right|}{1+\hbar \alpha \beta} .
$$

If $\alpha^{2}, \beta^{2}>0$, they imply the existence of both minimal position and momentum uncertainties, given by

$$
\Delta x_{M}=\frac{\hbar \beta}{\sqrt{1+2 \hbar \alpha \beta}} \sim \hbar \beta(1-\hbar \alpha \beta), \quad \Delta p_{M}=\frac{\hbar \alpha}{\sqrt{1+2 \hbar \alpha \beta}} \sim \hbar \alpha(1-\hbar \alpha \beta) .
$$

The limit values may not be achieved by physical states. With the choice of parameters discussed in sect. 1 , the numerical value of the minimal uncertainties are the Planck length, $\Delta x_{M} \sim 10^{-33} \mathrm{~cm}$, and an extremely tiny momentum, $\Delta p_{M} \sim 10^{-62} \mathrm{~g}$.

No minimal uncertainties emerge instead if $\alpha^{2}, \beta^{2}<0$.

\subsection{Representation of position and momentum operators}

Extending the transformations (2.5) to the quantum case, it is possible to define a realization of the position and momentum operators $\hat{x}_{i}$ and $\hat{p}_{i}$ that obeys the algebra (3.1). More precisely, the operators act on a Hilbert space of functions of a variable $P_{i}$, as

$$
\begin{aligned}
& \hat{x}_{i}=\hat{\mathcal{X}}_{i}+\lambda \frac{\beta}{\alpha} \hat{\mathcal{P}}_{i}=i \sqrt{1-\beta^{2} P_{k}^{2}} \frac{\partial}{\partial P_{i}}+\lambda \frac{\beta}{\alpha} \frac{P_{i}}{\sqrt{1-\beta^{2} P_{k}^{2}}}, \\
& \hat{p}_{i}=-\frac{\alpha}{\beta} \hat{\mathcal{X}}_{i}+(1-\lambda) \hat{\mathcal{P}}_{i}=-i \frac{\alpha}{\beta} \sqrt{1-\beta^{2} P_{k}^{2}} \frac{\partial}{\partial P_{i}}+(1-\lambda) \frac{P_{i}}{\sqrt{1-\beta^{2} P_{k}^{2}}},
\end{aligned}
$$

If $\alpha^{2}, \beta^{2}>0$, the range of allowed values of $P_{i}$ is bounded by $P_{k}^{2}<1 / \beta^{2}$. If $\alpha^{2}, \beta^{2}<0$, instead, all real values of $P_{i}$ are allowed, but the upper bound of the eigenvalues of the operator $\left(\alpha \hat{x}_{k}+\beta \hat{p}_{k}\right)^{2}$ is 1 , as in the classical limit.

In the previous formulae, the realizations corresponding to different values of $\lambda$ are, at least formally, unitary equivalent. For example, in the case $\alpha^{2}, \beta^{2}>0$, they are related through the action of the operator $U=\left(1-\beta^{2} P_{k}^{2}\right)^{\frac{i \lambda}{2 \hbar \alpha \beta}}$, so that $\hat{x}_{i}(\lambda)=U^{\dagger} \hat{x}_{i}(0) U$, $\hat{p}_{i}(\lambda)=U^{\dagger} \hat{p}_{i}(0) U$. One is therefore free to choose the value of $\lambda$ without affecting the physical predictions of the theory, and hence in the following, unless explicitly stated, we shall set $\lambda$ to 0 . An exception will be the investigation of the harmonic oscillator, where a different choice is more convenient.

We now discuss in detail the action of the operators in the simple case of a particle in one dimension in the representation (3.6) with $\lambda=0$. When $\alpha^{2}, \beta^{2}>0$, in order to get symmetric operators, i.e.

$$
(\hat{p} \psi, \phi)=(\psi, \hat{p} \phi), \quad(\hat{x} \psi, \phi)=(\psi, \hat{x} \phi),
$$

the scalar product must be defined as

$$
(\psi, \phi)=\int_{-1 / \beta}^{1 / \beta} \frac{d P}{\sqrt{1-\beta^{2} P^{2}}} \psi^{*}(P) \phi(P),
$$


and the wave functions must satisfy periodic boundary conditions, $\psi(-1 / \beta)=\psi(1 / \beta)$. This is readily checked like in ref. [16].

If instead $\alpha^{2}, \beta^{2}<0$, the scalar product is defined as

$$
(\psi, \phi)=\int_{-\infty}^{\infty} \frac{d P}{\sqrt{1-\beta^{2} P^{2}}} \psi^{*}(P) \phi(P),
$$

and only the convergence of the integral at infinity is required.

The study of the spectrum of the position and momentum operators encounters some difficulties. For example, the eigenvalue equation

$$
\hat{x} \psi_{x}=x \psi_{x}
$$

has solutions

$$
\psi_{x}=C \mathrm{e}^{-\frac{i x}{\hbar \beta} \arcsin \beta P},
$$

with $C$ a normalization constant and $x=2 n \hbar \beta$. The eigenstates (3.11) have vanishing position uncertainty, but their energy diverges, so that they cannot be accepted as physical. The same situation occurs in ordinary quantum mechanics, but in that case the position eigenstates can be obtained as limit of states with finite energy. This is not possible in our case, due to the existence of a minimal indetermination for the positions, which implies that no exactly localized states can exist. As in similar cases [18], one may try to define states with minimal position uncertainty by suitably modifying the equation that defines the position eigenstates, in order to take into account the finite uncertainty in position. However, we were not able to find a suitable modification. Nevertheless, since $[\hat{x}, \hat{p}]=[\hat{\mathcal{X}}, \hat{\mathcal{P}}]$ one may guess that the functions that minimize the commutator expectation value are the same as those that give rise to minimal uncertainty in flat Snyder space, i.e.

$$
\psi_{x}=C \sqrt{1-\beta^{2} P^{2}} \mathrm{e}^{-\frac{i x}{\hbar \beta} \arcsin \beta P} .
$$

Notice that the functions (3.12) satisfy the boundary conditions without need of quantizing the parameter $x$. For these functions,

$$
\Delta x=\hbar \beta, \quad \Delta p=\sqrt{\frac{1}{\beta^{2}}+\hbar^{2} \alpha^{2}}, \quad \Delta x \Delta p=\hbar \sqrt{1+(\hbar \alpha \beta)^{2}},
$$

and $\Delta x$ is close to its minimal value (3.5), while $\Delta p$ is extremely large, due to the smallness of $\beta$.

More general functions in the domain of $\hat{x}$ and $\hat{p}$ are given by

$$
\psi_{x}=C\left(1-\beta^{2} P^{2}\right)^{\rho} \quad \mathrm{e}^{-\frac{i x}{\hbar \beta} \arcsin \beta P},
$$

with $\operatorname{Re} \rho>\frac{1}{4}$, but these give rise to greater uncertainties. For example, for $\rho=\frac{1}{2}+i \mu$, one has

$$
(\Delta x)^{2}=\hbar^{2} \beta^{2}+\frac{\mu^{2}}{\alpha^{2}}, \quad(\Delta p)^{2}=\hbar^{2} \alpha^{2}+\frac{(1-\mu)^{2}}{\beta^{2}} .
$$


We notice that the value of $\Delta x \Delta p$ can be minimized for this class of functions by the choice $\mu=\left(1 \pm \sqrt{1-4 \hbar^{2} \alpha^{2} \beta^{2}}\right) / 2$, for which $\Delta x \Delta p=\hbar$.

The properties of the momentum operator $\hat{p}$ are similar to those of $\hat{x}$. The eigenvalue equation

$$
\hat{p} \psi_{p}=p \psi_{p},
$$

has solution

$$
\psi_{p}=C\left(1-\beta^{2} P^{2}\right)^{\frac{i}{2 \hbar \alpha \beta}} \mathrm{e}^{\frac{i p}{\hbar \alpha} \arcsin \beta P},
$$

with $p=2 n \hbar \alpha$, and presents problems analogous to those affecting the eigenfunctions of the position operator. In this case $\Delta p=0$, but the expectation value of $\hat{x}^{2}$ diverges. As before, however, one can define a basis, analogous to (3.12),

$$
\psi_{p}=C\left(1-\beta^{2} P^{2}\right)^{\frac{1}{2}+\frac{i}{2 \hbar \alpha \beta}} \mathrm{e}^{\frac{i p}{\hbar \alpha} \arcsin \beta P},
$$

where $p$ is not quantized, for which the expectation value of $\hat{x}^{2}$ is finite. The uncertainties in this basis are

$$
\Delta x=\sqrt{\frac{1}{\alpha^{2}}+\hbar^{2} \beta^{2}}, \quad \Delta p=\hbar \alpha, \quad \Delta x \Delta p=\hbar \sqrt{1+(\hbar \alpha \beta)^{2}} .
$$

More general functions analogous to (3.14) may also be considered.

To summarize, although formal eigenstates (3.11), (3.17) give rise to a discrete spectrum for both position and momentum, they are not physical because have infinite expectation value of $\hat{p}^{2}$ or $\hat{x}^{2}$. A more physical basis of states is given by smeared functions like (3.14), (3.18), that describe a fuzzy phase space, with no sharply defined values of positions and momenta.

In the aSdS case, the situation is instead analogous to ordinary quantum mechanics, since no minimal indetermination arises, and the formal eigenfunctions are limit of states with finite $\Delta x$ and $\Delta p$. For the position operator, the eigenfunction are

$$
\psi_{x}=C \mathrm{e}^{-\frac{i x}{\hbar \beta} \operatorname{arcsinh} \beta P},
$$

and for momentum,

$$
\psi_{p}=C\left(1-\beta^{2} P^{2}\right)^{\frac{i}{2 \hbar \alpha \beta}} \mathrm{e}^{\frac{i p}{\hbar \alpha} \operatorname{arcsinh} \beta P} .
$$

\subsection{Quantum symmetries}

The invariance of the configuration space of the classical model under the $S O(4)$ or $S O(3,1)$ group can be extended to the quantum case.

The rotations are generated by

$$
\hat{J}_{i j}=\frac{1}{2}\left(\hat{x}_{i} \hat{p}_{j}+\hat{p}_{j} \hat{x}_{i}-\hat{x}_{j} \hat{p}_{i}-\hat{p}_{i} \hat{x}_{j}\right)=\hat{\mathcal{P}}_{j} \hat{\mathcal{X}}_{i}-\hat{\mathcal{P}}_{i} \hat{\mathcal{X}}_{j}=i \hbar\left(P_{j} \frac{\partial}{\partial P_{i}}-P_{i} \frac{\partial}{\partial P_{j}}\right) .
$$


and act in the standard way. In particular, the spectrum of $\hat{J}_{i j}$ is the same as in ordinary quantum mechanics. Defining $\hat{L}_{i}=\epsilon_{i j k} \hat{J}_{j k}$, the eigenfunctions in spherical coordinates in momentum representation are given by the standard spherical harmonics

$$
\hat{L}^{2} Y_{l m}\left(P_{\theta}, P_{\phi}\right)=l(l+1) Y_{l m}\left(P_{\theta}, P_{\phi}\right), \quad \hat{L}_{z} Y_{l m}\left(P_{\theta}, P_{\phi}\right)=m Y_{l m}\left(P_{\theta}, P_{\phi}\right) \text {. }
$$

The translations are generated by the momentum operators $\hat{p}_{i}$, that act according to (3.1). While the action of the translations on momenta is the usual one for a space of constant curvature, that on position variables is deformed and takes a nonlinear form. The commutation relations (3.1) transform covariantly under these symmetries.

\section{QUANTUM DYNAMICS IN ONE DIMENSION}

We shall now use the results of the previous section to study the solutions of some simple one-dimensional quantum systems. We write down in detail the calculations for the SdS model, and simply report the results for the aSdS case, that can be investigated in the same way.

\subsection{Free particle}

Let us first consider the Schrödinger equation for a free particle in one dimension. In the representation (3.6) with $\lambda=0$, for unit mass it reads

$$
\frac{d^{2} \psi}{d P^{2}}-\left(\beta-\frac{2 i}{\hbar \alpha}\right) \frac{\beta P}{1-\beta^{2} P^{2}} \frac{d \psi}{d P}-\frac{\beta^{2}}{\hbar^{2} \alpha^{2}}\left[\frac{P^{2}-i \hbar \alpha / \beta}{\left(1-\beta^{2} P^{2}\right)^{2}}-\frac{2 E}{1-\beta^{2} P^{2}}\right] \psi=0
$$

In the SdS case, solutions of (4.1) that vanish at $P= \pm 1 / \beta$ exist, of the form

$$
\psi=\text { const. } \times\left(1-\beta^{2} P^{2}\right)^{\frac{i}{2 \hbar \alpha \beta}} \cos \left[\frac{\sqrt{2 E}}{\hbar \alpha} \arcsin \beta P\right],
$$

with $E=\frac{1}{2} \hbar^{2} \alpha^{2} n^{2}$, for odd integer $n$. These solutions have finite values of $\Delta x$.

For aSdS, the relevant solutions are instead given by the momentum eigenfunctions (3.21), and the energy is not quantized.

\subsection{Harmonic oscillator}

Let us now consider the one-dimensional quantum harmonic oscillator, with Hamiltonian

$$
H=\frac{\hat{p}^{2}}{2 m}+\frac{m \omega_{0}^{2} \hat{x}^{2}}{2} .
$$

In order to simplify the calculations, we exploit the possibility of choosing the coefficient $\lambda$ in the representation (3.6) so that the cross terms $\hat{\mathcal{P}} \hat{\mathcal{X}}+\hat{\mathcal{X}} \hat{\mathcal{P}}$ in the Hamiltonian vanish, setting

$$
\lambda=\frac{\alpha^{2}}{\beta^{2} \omega_{0}^{2}+\alpha^{2}}
$$


With this choice, the Schrödinger equation reads, for unit mass,

$$
\frac{1}{2} \frac{\beta^{2} \omega_{0}^{2}}{\beta^{2} \omega_{0}^{2}+\alpha^{2}}\left[\hat{\mathcal{P}}^{2}+\frac{\left(\beta^{2} \omega_{0}^{2}+\alpha^{2}\right)^{2}}{\beta^{4} \omega_{0}^{2}} \hat{\mathcal{X}}^{2}\right] \psi=E \psi
$$

Using the realization (3.6) of the operators, eq. (4.5) can be written as

$$
\frac{d^{2} \psi}{d P^{2}}-\frac{\beta^{2} P}{1-\beta^{2} P^{2}} \frac{d \psi}{d P}-\frac{1}{\hbar^{2} \omega^{2}}\left[\frac{P^{2}}{\left(1-\beta^{2} P^{2}\right)^{2}}-\frac{2 \mathcal{E}}{1-\beta^{2} P^{2}}\right] \psi=0,
$$

where $\omega=\left(1+\frac{\alpha^{2}}{\beta^{2} \omega_{0}^{2}}\right) \omega_{0}$ and $\mathcal{E}=\left(1+\frac{\alpha^{2}}{\beta^{2} \omega_{0}^{2}}\right) E$.

The form of this equation is the same that holds for the flat Snyder model [16], but with different coefficients, and can be solved in the same way. In particular, when $\alpha^{2}, \beta^{2}>0$, one can define a variable $\bar{P}=\arcsin \beta P$, in terms of which (4.6) becomes the standard Schrödinger equation for a potential

$$
V=\frac{1}{\omega^{2}} \tan ^{2} \bar{P}
$$

that coincides with the classical potential of sect. 2 .

In order to find the explicit solution of eq. (4.6), it is more convenient, however, to define the variable $z=(1+\beta P) / 2$, in terms of which the equation can be written in the hypergeometric form

$$
\frac{d^{2} \psi}{d z^{2}}+\frac{z-\frac{1}{2}}{z(z-1)} \frac{d \psi}{d z}-\left[\frac{\mu\left(z-\frac{1}{2}\right)^{2}}{z^{2}(z-1)^{2}}+\frac{\epsilon}{z(z-1)}\right] \psi=0
$$

with

$$
\mu=\frac{\omega_{0}^{2}}{\hbar^{2}\left(\beta^{2} \omega_{0}^{2}+\alpha^{2}\right)^{2}}, \quad \epsilon=\frac{2 E}{\hbar^{2}\left(\beta^{2} \omega_{0}^{2}+\alpha^{2}\right)} .
$$

By standard methods, one can then obtain the solution in terms of the hypergeometric function $\mathbf{F}(a, b, c ; z)$,

$$
\psi=\text { const } \times\left(1-\beta^{2} P^{2}\right)^{(1+\sqrt{1+4 \mu}) / 4} \mathbf{F}\left(a, b, c ; \frac{1+\beta P}{2}\right),
$$

where

$$
a=\frac{1}{2}(1+\sqrt{1+4 \mu})-\sqrt{\mu+\epsilon}, \quad b=\frac{1}{2}(1+\sqrt{1+4 \mu})+\sqrt{\mu+\epsilon}, \quad c=1+\frac{1}{2} \sqrt{1+4 \mu} .
$$

We require that $\psi$ vanish at $P= \pm 1 / \beta$, i.e. at $z=0,1$. This occurs when $a=-n$ or $b=-n$. In both cases,

$$
\epsilon=\left(n+\frac{1}{2}\right)(1+\sqrt{1+4 \mu})+n^{2},
$$


and the solution can be written in terms of Gegenbauer polynomials $\mathbf{C}_{n}^{\alpha}[20]$ as

$$
\psi=\text { const } \times\left(1-\beta^{2} P^{2}\right)^{\alpha / 2} \mathbf{C}_{n}^{\alpha}(\beta P),
$$

with $\alpha=\frac{1}{2}(1+\sqrt{1+4 \mu})$.

From (4.10) follows the spectrum of the energy,

$$
E=\left(n+\frac{1}{2}\right) \hbar \omega_{0} \sqrt{1+\frac{\hbar^{2}\left(\beta^{2} \omega_{0}^{2}+\alpha^{2}\right)^{2}}{4 \omega_{0}^{2}}}+\left(n^{2}+n+\frac{1}{2}\right) \frac{\hbar^{2}\left(\beta^{2} \omega_{0}^{2}+\alpha^{2}\right)}{2}
$$

which exhibits corrections of order $\hbar\left(\beta^{2} \omega_{0}+\alpha^{2} / \omega_{0}\right)$ with respect to the standard case and a duality for $\beta^{2} \omega_{0} \leftrightarrow \alpha^{2} / \omega_{0}$.

In the limit $\alpha \rightarrow 0$ one recovers the results of [16] for flat Snyder space, whereas for $\beta \rightarrow 0$, one obtains the energy spectrum on a 3 -sphere,

$$
E=\left(n+\frac{1}{2}\right) \hbar \omega_{0} \sqrt{1+\frac{\hbar^{2} \alpha^{4}}{4 \omega_{0}^{2}}}+\left(n^{2}+n+\frac{1}{2}\right) \frac{\hbar^{2} \alpha^{2}}{2}
$$

for which at first order the shift in the energy with respect to the standard oscillator is independent of $\omega_{0}$.

The same calculation can be performed when $\alpha^{2}, \beta^{2}<0$. The energy spectrum is simply the analytic continuation of (4.12) for negative values of $\alpha^{2}$ and $\beta^{2}$. In this case, for great $n$ the energy becomes negative. In order to ensure positivity of energy, one must therefore impose an upper bound on the allowed values of $n$.

\section{QUANTUM DYNAMICS IN THREE DIMENSIONS}

Of course, the extension of the previous results to three-dimensional space is of great physical relevance, since it permits to test the effect of the noncommutativity of spatial coordinates.

The analysis of the previous sections easily generalizes to three dimensions. Since the components of the position and momentum operators $\hat{x}_{i}$ and $\hat{p}_{i}$ do not commute, we shall limit our consideration to spherically symmetric problems, where one can use polar coordinates, that much simplify the problem. Moreover, we set $\hbar=1$ in this section.

\subsection{Position eigenstates in three dimensions}

As in standard quantum mechanics, a basis of operators for one-particle states in momentum representation is given by the radial component of the momentum $\hat{p}_{r}$ and by the angular momentum $\hat{L}_{i}$, introduced in section 3.3.

Therefore, we adapt the representation (3.6) to three-dimensional spherical coordinates in the $P_{i}$-space. We first define the operators

$$
\hat{\mathcal{P}}_{r}=\mathcal{P}_{r} \equiv \sqrt{\mathcal{P}_{i}^{2}}=\sqrt{\frac{P_{i}^{2}}{1-\beta^{2} P_{i}^{2}}}, \quad \hat{\mathcal{X}}_{r}=\sqrt{1-\beta^{2} P_{r}^{2}}\left(i \frac{\partial}{\partial P_{r}}+\frac{1}{P_{r}}\right)
$$


with $\left[\hat{\mathcal{X}}_{r}, \hat{\mathcal{P}}_{r}\right]=i$. As in ordinary quantum mechanics it follows that

$$
\hat{\mathcal{P}}_{i}^{2}=\hat{\mathcal{P}}_{r}^{2}, \quad \hat{\mathcal{X}}_{i}^{2}=\hat{\mathcal{X}}_{r}^{2}+\frac{\hat{L}^{2}}{\mathcal{P}_{r}^{2}}, \quad \hat{\mathcal{X}}_{i} \hat{\mathcal{P}}_{i}+\hat{\mathcal{P}}_{i} \hat{\mathcal{X}}_{i}=\hat{\mathcal{X}}_{r} \hat{\mathcal{P}}_{r}+\hat{\mathcal{P}}_{r} \hat{\mathcal{X}}_{r},
$$

where $\hat{L}^{2}$ is the square of the angular momentum operator. The square of the position and momentum operators (3.6) can then be written in terms of the radial operators as

$$
\hat{x}_{i}^{2}=\left(\hat{\mathcal{X}}_{r}+\frac{\beta}{\alpha} \lambda \hat{\mathcal{P}}_{r}\right)^{2}+\frac{\hat{L}^{2}}{\mathcal{P}_{r}^{2}}, \quad \hat{p}_{i}^{2}=\left((1-\lambda) \hat{\mathcal{P}}_{r}-\frac{\alpha}{\beta} \hat{\mathcal{X}}_{r}\right)^{2}+\frac{\alpha^{2}}{\beta^{2}} \frac{\hat{L}^{2}}{\mathcal{P}_{r}^{2}}
$$

Since the action of the angular momentum is the standard one, as discussed in section 3.3, a generic three-dimensional wave function can be expanded in spherical harmonics, as defined in (3.23),

$$
\psi\left(P_{r}, P_{\theta}, P_{\phi}\right)=\sum_{l, m} \psi_{r l m}\left(P_{r}\right) Y_{l m}\left(P_{\theta}, P_{\phi}\right)
$$

and only the radial functions need to be investigated in detail. In the following we shall omit the $l m$ indices in the radial functions.

The scalar product in the space of the radial functions can be defined as

$$
\left(\psi_{r}, \phi_{r}\right)=\int_{0}^{1 / \beta} \frac{P_{r}^{2} d P_{r}}{\sqrt{1-\beta^{2} P_{r}^{2}}} \psi_{r}^{*}\left(P_{r}\right) \phi_{r}\left(P_{r}\right)
$$

The spectrum of the radial momentum and position operators will be analogous to the one of the corresponding one-dimensional operators, except that now $P_{r}$ can only take positive values. Therefore we shall not discuss it in detail, but rather pass to investigate the Schrödinger equation.

The basis adopted in this section also permits to immediately single out the states that minimize the uncertainty relations between the position coordinates along different directions. In fact, from (3.1) it follows that

$$
\Delta x_{i} \Delta x_{j} \geq\left|\frac{\beta^{2}}{2}\left\langle\hat{J}_{i j}\right\rangle\right|
$$

and the states that minimize these uncertainty relations are those with vanishing angular momentum. The same considerations hold for the components of the momentum.

The previous discussion can easily be extended to the anti-Snyder model.

\subsection{The free particle in three dimensions}

Let us consider the Schrödinger equation for a free particle in three dimensions. Choosing the gauge $\lambda=0$, and using the representation (5.1), (5.3) and the expansion (5.4) in spherical harmonics, the radial part of the Schrödinger equation can be written as

$$
\begin{aligned}
\frac{d^{2} \psi_{r}}{d P_{r}^{2}} & -\frac{\left(3 \beta^{2}-2 i \frac{\beta}{\alpha}\right) P_{r}^{2}-2}{P_{r}\left(1-\beta^{2} P_{r}^{2}\right)} \frac{d \psi_{r}}{d P_{r}} \\
& -\frac{\beta^{2}}{\alpha^{2}}\left[\frac{(1-4 i \alpha \beta) P_{r}^{2}+3 i \frac{\alpha}{\beta}}{\left(1-\beta^{2} P_{r}^{2}\right)^{2}}+\frac{l(l+1) \alpha^{2}}{\beta^{2} P_{r}^{2}}-\frac{2 E}{1-\beta^{2} P_{r}^{2}}\right] \psi_{r}=0 .
\end{aligned}
$$


Defining now a function $u\left(P_{r}\right)$ such that $\psi_{r}=\left(1-\beta^{2} P_{r}^{2}\right)^{i / 2 \alpha \beta} u$, eq. (5.7) simplifies to

$$
\frac{d^{2} u}{d P_{r}^{2}}+\left(\frac{2}{P_{r}}-\frac{\beta^{2} P_{r}}{1-\beta^{2} P_{r}^{2}}\right) \frac{d u}{d P_{r}}-\left[\frac{l(l+1)}{P_{r}^{2}}-\frac{2 \beta^{2} E}{\alpha^{2}\left(1-\beta^{2} P_{r}^{2}\right)}\right] u=0 .
$$

Finally, after a change of variable $z=\beta^{2} P_{r}^{2}$, the equation takes the form of a hypergeometric differential equation,

$$
\frac{d^{2} u}{d z^{2}}+\frac{3-4 z}{2 z(1-z)} \frac{d u}{d z}-\frac{1}{4}\left[\frac{l(l+1)}{z^{2}}-\frac{\epsilon}{z(1-z)}\right] u=0,
$$

where $\epsilon=2 E / \alpha^{2}$, with solution

$$
u\left(P_{r}\right)=\text { const } \times \sqrt{1-\beta^{2} P_{r}^{2}}\left(\beta P_{r}\right)^{l} \mathbf{F}\left(a, b, c ; \beta^{2} P_{r}^{2}\right),
$$

with $\mathbf{F}$ a hypergeometric function of parameters

$$
a=1+\frac{l}{2}+\frac{\sqrt{1+\epsilon+l(l+1)}}{2}, \quad b=1+\frac{l}{2}-\frac{\sqrt{1+\epsilon+l(l+1)}}{2}, \quad c=l+\frac{3}{2} .
$$

The solution of (5.7) is therefore

$$
\psi_{r}=\left(1-\beta^{2} P_{r}^{2}\right)^{\frac{1}{2}(1+i / \alpha \beta)}\left(\beta P_{r}\right)^{l} \mathbf{F}\left(a, b, c ; \beta^{2} P_{r}^{2}\right)
$$

The boundary conditions must be fixed in such a way that $\psi_{r}$ vanish at $P_{r}=1 / \beta$, i.e. at $z=1$. This occurs when $b=-n$, with integer $n$. It follows that

$$
E=\alpha^{2}\left(2 n^{2}+4 n+2 n l+\frac{3}{2} l+\frac{3}{2}\right)
$$

Hence, in the SdS case, the eigenvalues of the energy are quantized. This is of course a consequence of the finite range of the coordinate $P_{r}$. The radial wave function can be written

$$
\psi_{r}=\text { const. } \times\left(1-\beta^{2} P_{r}^{2}\right)^{\frac{1}{2}(1+i / \alpha \beta)}\left(\beta P_{r}\right)^{l} \mathbf{P}_{n}^{\left(l+\frac{1}{2}, \frac{1}{2}\right)}\left(1-2 \beta^{2} P_{r}^{2}\right),
$$

with $\mathbf{P}_{n}^{(\mu, \nu)}$ a Jacobi polynomial [20].

For the spherical wave, $l=0$, the solution (5.12) takes a simple form. Using the properties of the hypergeometric functions, one easily gets

$$
\psi_{r 0}=\left(1-\beta^{2} P_{r}^{2}\right)^{i / 2 \alpha \beta} \frac{\sin \left[\sqrt{1+\epsilon} \arcsin \beta P_{r}\right]}{\sqrt{1+\epsilon} \beta P_{r}},
$$

that in the limit $\alpha \rightarrow 0, \beta \rightarrow 0$ coincides with the standard quantum-mechanical solution $\psi_{r 0}=\sin \left(\sqrt{2 E} P_{r}\right) /\left(\sqrt{2 E} P_{r}\right)$. 
In the aSdS case, the Schrödinger equation is the analytic continuation of (5.7) to negative values of $\alpha^{2}$ and $\beta^{2}$. Requiring regularity for $P_{r} \rightarrow \infty$, the solution reads

$$
\psi_{r}=\text { const. } \times \frac{\left(1-\beta^{2} P_{r}^{2}\right)^{i / 2 \alpha \beta}}{\left(\beta^{2} P_{r}^{2}\right)^{a}} \mathbf{F}\left(a, a-c+1, a-b+1, \frac{1}{\beta^{2} P_{r}^{2}}\right)
$$

and does not imply a quantization of the energy.

\subsection{The harmonic oscillator in three dimensions}

In order to write the Schrödinger equation for the 3-dimensional harmonic oscillator, one can proceed as for the free particle. However, in this case it is convenient to choose $\lambda$ as in (4.4), in order to remove the mixed terms from the equation. With this choice, one obtains

$$
\frac{1}{2} \frac{\beta^{2} \omega_{0}^{2}}{\beta^{2} \omega_{0}^{2}+\alpha^{2}}\left[\hat{\mathcal{P}}_{i}^{2}+\frac{\left(\beta^{2} \omega_{0}^{2}+\alpha^{2}\right)^{2}}{\beta^{4} \omega_{0}^{2}} \hat{\mathcal{X}}_{i}^{2}\right] \psi=E \psi .
$$

Expanding in spherical harmonics as in (5.4), after some algebraic manipulations the equation for the radial wave function becomes

$$
\begin{aligned}
\frac{d^{2} \psi_{r}}{d P_{r}^{2}} & +\left(\frac{2}{P_{r}}-\frac{\beta^{2} P_{r}}{1-\beta^{2} P_{r}^{2}}\right) \frac{d \psi_{r}}{d P_{r}} \\
& -\left[\frac{l(l+1)}{P_{r}^{2}}+\frac{1}{\omega^{2}} \frac{P_{r}^{2}}{\left(1-\beta^{2} P_{r}^{2}\right)^{2}}-\left(\frac{2 \mathcal{E}}{\omega^{2}}-\beta^{2}\right) \frac{1}{1-\beta^{2} P_{r}^{2}}\right] \psi_{r}=0
\end{aligned}
$$

where $\omega=\left(1+\frac{\alpha^{2}}{\beta^{2} \omega_{0}^{2}}\right) \omega_{0}$ and $\mathcal{E}=\left(1+\frac{\alpha^{2}}{\beta^{2} \omega_{0}^{2}}\right) E$.

Defining a new variable $z=\beta^{2} P_{r}^{2},(5.15)$ can be put in the form of a hypergeometric equation,

$$
\frac{d^{2} \psi_{r}}{d z^{2}}+\frac{3-4 z}{2 z(1-z)} \frac{d \psi_{r}}{d z}-\frac{1}{4}\left[\frac{l(l+1)}{z^{2}}+\frac{\mu}{(1-z)^{2}}-\frac{\epsilon}{z(1-z)}\right] \psi_{r}=0
$$

with

$$
\mu=\frac{1}{\beta^{4} \omega^{2}}, \quad \epsilon=\frac{2 \mathcal{E}}{\beta^{2} \omega^{2}}-1
$$

Note that (5.16) differs from the free particle equation (5.9) only for the term proportional to $\mu$. The solution of (5.16) can be written as

$$
\psi_{r}=\text { const } \times\left(\beta P_{r}\right)^{l}\left(1-\beta^{2} P_{r}^{2}\right)^{(1+\sqrt{1+4 \mu}) / 4} \mathbf{F}\left(a, b, c ; \beta^{2} P_{r}^{2}\right),
$$

with

$$
\begin{aligned}
& a=\frac{1}{2}\left(l+\frac{3}{2}+\frac{\sqrt{1+4 \mu}}{2}+\sqrt{1+\mu+\epsilon+l(l+1)}\right), \\
& b=\frac{1}{2}\left(l+\frac{3}{2}+\frac{\sqrt{1+4 \mu}}{2}-\sqrt{1+\mu+\epsilon+l(l+1)}\right), \\
& c=l+\frac{3}{2}
\end{aligned}
$$


We require that $\psi$ vanish at $P_{r}=1 / \beta$, i.e. at $z=1$. This occurs when $b=-n$, and then

$$
\epsilon=\left(2 n+l+\frac{3}{2}\right) \sqrt{1+4 \mu}+4 n^{2}+4 n l+6 n+2 l+\frac{3}{2},
$$

and

$$
\psi_{r}=\mathrm{const} \times\left(\beta P_{r}\right)^{l}\left(1-\beta^{2} P_{r}^{2}\right)^{(1+\sqrt{1+4 \mu}) / 4} \mathbf{P}_{n}^{\left(l+\frac{1}{2}, \frac{\sqrt{1+4 \mu}}{2}\right)}\left(1-2 \beta^{2} P_{r}^{2}\right),
$$

with $\mathbf{P}_{n}^{(\mu, \nu)}$ a Jacobi polynomial. From (5.18) it follows that

$$
E=\left(2 n+l+\frac{3}{2}\right) \omega_{0} \sqrt{1+\frac{\left(\beta^{2} \omega_{0}^{2}+\alpha^{2}\right)^{2}}{4 \omega_{0}^{2}}}+\left(2 n^{2}+3 n+2 n l+l+\frac{5}{4}\right)\left(\beta^{2} \omega_{0}^{2}+\alpha^{2}\right) .
$$

The previous expression may also be written in terms of a new quantum number $N=2 n+l$, that is commonly introduced for the three-dimensional oscillator,

$$
E=\left(N+\frac{3}{2}\right) \omega_{0} \sqrt{1+\frac{\left(\beta^{2} \omega_{0}^{2}+\alpha^{2}\right)^{2}}{4 \omega_{0}^{2}}}+\left(N^{2}+3 N-l(l+1)+\frac{5}{2}\right) \frac{\beta^{2} \omega_{0}^{2}+\alpha^{2}}{2} .
$$

Like in one dimension, corrections of order $\left(\beta^{2} \omega_{0}+\alpha^{2} / \omega_{0}\right)$ with respect to ordinary quantum mechanics occur in the spectrum of the harmonic oscillator. In the limit $\alpha \rightarrow 0$ one recovers the result valid for flat Snyder space, whereas for $\beta \rightarrow 0$, one obtains the spectrum of the oscillator on a 3 -sphere. In this case, at first order the shift in the energy with respect to the standard oscillator is independent of $\omega_{0}$.

The energy spectrum of the 3-dimensional aSdS harmonic oscillator can be obtained by analytic continuation of that of the SdS oscillator to negative $\alpha^{2}$ and $\beta^{2}$, and hence is still given by (5.20). As in one dimension, one must impose some conditions on the quantum numbers in order to preserve the positivity of the energy.

\section{CONCLUSIONS}

We have studied the classical and the quantum mechanics of the free particle and of the harmonic oscillator in the nonrelativistic version of the Snyder model on a constant curvature background. The calculations have been based on the existence of a linear mapping relating the (a)SdS phase space variables to flat Snyder coordinates.

As in the flat case, the physics strongly depends on the sign of the coupling constants in the defining Poisson brackets (or commutation relations in the quantum case). In particular, we have been able to construct the Snyder model on a space of constant positive curvature, and the anti-Snyder model on a space of constant negative curvature. In both cases, the curvature of momentum space has the same sign as that of position space.

In the SdS case, a bound on the localization of particles in both position and momentum space arises, while this does not occur in aSdS. Moreover, contrary to the flat anti-Snyder model, the range of values of the momentum in aSdS is not limited, although 
an upper bound holds for a linear combination of the position and momentum of a particle. This can be interpreted as an example of momentum-dependent geometry, like in other generalizations of DSR to curved spaces $[19,14]$. Even if we have not discusseed this topic, one can also employ the same arguments used for a flat background [16], to show that quantization of area arises for SdS, but not for aSdS.

In the limit $\alpha \rightarrow 0$, one recovers the results valid for flat Snyder space, while for $\beta \rightarrow 0$, one obtains the dynamics on a space of constant curvature. The study of this limit may also be useful in the relativistic case for the investigation of motion in (anti)-de Sitter spacetime.

The classical dynamics of free particles does not give rise to particularly relevant effects, while in the quantum case the energy spectrum is discrete for SdS and continuous for aSdS. The behavior of the one-dimensional harmonic oscillator is more interesting: both in the classical and in the quantum cases, its frequency depends on the energy. Moreover, in aSdS the quantum energy spectrum has only a finite number of eigenvalues.

The three-dimensional dynamics is similar to that governing the one-dimensional case, and can be investigated by means of an expansion in spherical harmonics, exploiting the fact that the angular momentum operator in (a)SdS enjoys standard properties. Its interest relies on the fact that the effects of the noncommutativity of spacetime and momentum space can be displayed explicitly. It turns out that minimal indeterminations are obtained for states with vanishing angular momentum.

Another relevant result is obtained in the limit $\beta \rightarrow 0$, that, as mentioned above, gives the dynamics on a 3-sphere. To our knowledge, no exact solutions of the quantum harmonic oscillator on a 3-sphere have previously been discussed in the literature.

It would be interesting to extend our results to more general systems, like for example the Coulomb potential. However, when the potential is nonpolynomial problems arise because of the nonlocality of the Hamiltonian when our transformation is applied, and only approximate solutions can be found.

An alternative approach to the problem studied in this paper, analogous to that adopted in [17] for the Snyder model, would consist in the investigation of the representations of the symmetry group $S O(5)$ on the coset space $S O(5) /(S O(3) \times S O(2))$, which is the phase space of three-dimensional Euclidean $\mathrm{SdS}$, or of the group $S O(4,1)$ on $S O(4,1) /(S O(2,1) \times S O(2))$ for aSdS.

Our study is limited to a nonrelativistic curved background. However, it can be used as a starting point to extend our results to the relativistic case and to quantum field theory. This would be important in the context of quantum gravity, where noncommutative curved spacetimes are expected to play a crucial role. This topic is presently under investigation.

\section{Acknowledgements}

I wish to thank M. Stetsko for pointing out an error in a previous version of this paper. 


\section{References}

[1] H.S. Snyder, Phys. Rev. 71, 38 (1947).

[2] S. Majid and H. Ruegg, Phys. Lett. B329, 189 (1994); S. Doplicher, K. Fredenhagen and J.E. Roberts, Phys. Lett. B331, 39 (1994).

[3] A. Kempf, Lett. Math. Phys. 26, 1 (1992); M. Maggiore, Phys. Lett. B304, 63 (1993).

[4] T. Padmanhaban, Ann. Phys. 165, 38 (1985); G. Veneziano, Europhys. Lett. 2, 199 (1986).

[5] C.A. Mead, Phys. Rev. 135, B849 (1964).

[6] G. Amelino-Camelia, Phys. Lett. B510, 255 (2001), Int. J. Mod. Phys. D11, 35 (2002); J. Magueijo and L. Smolin, Phys. Rev. Lett. 88, 190403 (2002).

[7] J. Kowalski-Glikman and S. Nowak, Int. J. Mod. Phys. D13, 299 (2003).

[8] S. Mignemi, Phys. Lett. B672, 186 (2009).

[9] E.J. Hellund and K. Tanaka, Phys. Rev. 94, 192 (1954); G. Jaroszkiewicz, J. Phys. A28, L343 (1995); J.M. Romero and A. Zamora, Phys. Rev. D70, 105006 (2004); E.R. Livine and D. Oriti, JHEP 0406, 050 (2004); R. Banerjee, S. Kulkarni and S. Samanta, JHEP 0605, 077 (2006); M.V. Battisti and S. Meljanac, Phys. Rev. D79, 067505 (2009).

[10] J. Kowalski-Glikman and L. Smolin, Phys. Rev. D70, 065020 (2004).

[11] C. Chryssomakolos and E. Okon, Int. J. Mod. Phys. D13, 1817 (2004).

[12] C.N. Yang, Phys. Rev. 72, 874 (1947).

[13] S. Mignemi, Class. Quantum Grav. 26, 245020 (2009).

[14] S. Mignemi, Annalen der Physik 522, 924 (2010).

[15] H.G. Guo, C.G. Huang and H.T. Wu, Phys. Lett. B663, 270 (2008); M.C. Carrisi and S. Mignemi, Phys. Rev. D82, 105031 (2010); R. Banerjee, K. Kumar and D. Roychowdhury, JHEP 1103, 060 (2011).

[16] S. Mignemi, Phys. Rev. D84, 025021 (2011).

[17] Lei Lu and A. Stern, arXiv:1108.1832.

[18] A. Kempf, G. Mangano and R.B. Mann, Phys. Rev. D52, 1108 (1995).

[19] J. Magueijo and L. Smolin, Class. Quantum Grav. 21, 1725 (2004).

[20] M. Abramowitz and I.A. Stegun, Handbook of mathematical fuctions, Dover 1965. 